\title{
Real alternative to quantum gravity in loop space
}

\author{
R. Loll \\ Sezione INFN di Firenze, Largo E. Fermi 2, I-50125 Firenze, Italy
}

(Received 20 February 1996)

\begin{abstract}
We show that the Hamiltonian constraint of four-dimensional Lorentzian gravity, defined on a space of real, SU(2)-valued connections, in spite of its nonpolynomiality possesses a natural quantum analogue in a latticediscretized formulation of the theory. This opens the way for a systematic search of its zero eigenvectors. The unambiguous and well-defined kinematical scalar product is that of the SU(2)-gauge theory. We also comment on various aspects of the continuum theory. [S0556-2821(96)01818-8]
\end{abstract}

PACS number(s): 04.60.Ds, 04.60.Nc

In spite of considerable advances in our understanding of the canonical quantization of gravity, based on the use of complex connection variables [1] and quantum representations on spaces of Wilson loops [2], some basic problems have remained unsolved. First, despite the apparent simplicity and polynomiality of the Hamiltonian in this approach, even at a formal level, only a few (and for the most part physically uninteresting) solutions to the Wheeler-DeWitt equation have been found in any loop representation. Second, treatment of the reality conditions (which have to be imposed on the $\operatorname{SL}(2, \mathbb{C})$-valued basic variables to recover real Einsteinian gravity) continues to be troublesome in the quantum theory. [The heat kernel measure on $\mathrm{SL}(2, \mathscr{C})$-wave functions used in $[3,4]$ provides a kinematic scalar product for complex Wilson loops (modulo a possible hidden metric dependence), but does not incorporate correctly the reality conditions for Ashtekar gravity.]

Faced with these difficulties, it may be time to remember that there exists a version of Hamiltonian gravity in terms of real connection variables [5] (for a corresponding action principle, see [6]), and to reevaluate the achievements and drawbacks of the complex formulation as compared to this real alternative. There is a "unified" derivation of the two connection representations in the classical theory: starting from the $3+1$ formulation in terms of a canonically conjugate pair $\left(P_{i}^{a}, K_{a}^{i}\right)$ of $\mathrm{SO}(3)$-valued variables, where $P_{i}^{a}$ denotes a dreibein (with density weight 1) and $K_{a}^{i}=(1 / \sqrt{g}) K_{a b} P^{b i}$ is (whenever the Gauss law constraints are satisfied) the extrinsic curvature with one index raised, one may define a canonical transformation

$$
\begin{gathered}
E_{i}^{a}=\alpha P_{i}^{a}, \\
A_{a}^{i}=\Gamma_{a}^{i}+\beta K_{a}^{i},
\end{gathered}
$$

where $\Gamma \equiv \Gamma(P)$ is the spin connection compatible with $P$, and $\alpha$ and $\beta$ are two nonvanishing constants. In terms of the new variables, one has

$$
\begin{aligned}
\left\{A_{a}^{i}(x), E_{j}^{b}(y)\right\} & =\alpha \beta\left\{K_{a}^{i}(x), P_{j}^{b}(y)\right\} \\
& =-\alpha \beta \delta_{j}^{i} \delta_{a}^{b} \delta^{3}(x-y) .
\end{aligned}
$$

The Hamiltonian constraint function in terms of $(A, E)$ reads

$$
\epsilon^{i j k} E_{i}^{a} E_{j}^{b} F_{a b}^{k}-\left[\left(2 / \beta^{2}+2\right)\right] E_{i}^{[a} E_{j}^{b]}\left(A_{a}^{i}-\Gamma_{a}^{i}\right)\left(A_{b}^{j}-\Gamma_{b}^{j}\right) .
$$

Ashtekar's choice is $\alpha=1, \beta=-i$, which makes the second, nonpolynomial term drop out of the Hamiltonian. The drawback is that, according to formula (1), the connection variable $A_{a}^{i}$ is now complex. Our real version of connection gravity consists in choosing $\alpha=1$ and $\beta=-1$ [5]. The functional forms for the remaining spatial diffeomorphism constraints $E_{k}^{a} F_{a b}^{k}=0$ and Gauss law constraints $\nabla_{a} E_{i}^{a}=0$ are independent of the choice of $\alpha$ and $\beta$. (As usual, we denote the field strength of the connection $A_{a}^{i}$ by $F$ and its covariant derivative by $\nabla$.) Their treatment therefore does not have to be changed with respect to the usual, complex formulation. In particular, we will continue to use Wilson loop variables in the quantum theory. In the real formulation, Eq. (2) becomes

$$
H^{\mathrm{R}}=\epsilon^{i j k} E_{i}^{a} E_{j}^{b} F_{a b}^{k}-H^{\mathrm{pot}},
$$

retaining a "potential" term (which is a misnomer since it depends both on coordinates and momenta). Starting from a form equivalent to Eq. (2) [formula (14) in Barbero's paper [5]] for $H^{\text {pot }}$, one finds after some algebra that it may be reexpressed as a polynomial in $A$ and $E$, up to determinantal factors, namely,

$$
\begin{aligned}
H^{\mathrm{pot}}= & (\operatorname{det} E)^{-2} \eta_{a_{1} a_{3} a_{4}} \eta_{b_{1} b_{3} b_{4}}\left(E_{k}^{a_{3}} E_{l}^{a_{4}} E_{m}^{b_{3}} E_{n}^{b_{4}}\right. \\
& \left.-2 E_{m}^{a_{3}} E_{n}^{a_{4}} E_{k}^{b_{3}} E_{l}^{b_{4}}\right) E_{k}^{a_{2}} E_{m}^{b_{2}}\left(\nabla_{a_{2}} E_{l}^{a_{1}}\right)\left(\nabla_{b_{2}} E_{n}^{b_{1}}\right),
\end{aligned}
$$

and up to terms proportional to the Gauss law constraints.

Let us now recall some features of the complex formulation with the Hamiltonian $H^{\mathbb{C}}=\epsilon^{i j k} E_{i}^{a} E_{j}^{b} F_{a b}^{k}$. There exist simple solutions to $\hat{H}^{\mathbb{C}} \Psi=0$ in the loop representation, both in the formal continuum approach [2] and on the lattice [4], where the loop state $\Psi$ depends on (smooth) nonintersecting loops (see also [7] for a generalization within a lattice language). This is a straightforward consequence of the antisymmetry of $H^{\mathbb{C}}$ in the spatial indices $a$ and $b$. However, these solutions are probably not interesting from a physical point of view, because they correspond to zero eigenstates of the volume operator [8,9]. Apart from the state found in [10], we are not aware of any nontrivial (in this sense) solutions that have been found by tackling the equation $\hat{H}^{\mathbb{C}} \Psi=0$ directly.

Other interesting features of the complex formulation are that all four diffeomorphism constraints can be solved by making a so-called Capovilla-Dell-Jacobson (CDJ) ansatz 
[11], and that there exists a formal solution to the WheelerDeWitt equation (with a specific factor ordering for $\hat{H}^{\mathbb{C}}$ ), given by the exponential of the Chern-Simons action for the complex connection $A$ [12]. However, in the absence of a proper treatment of the reality conditions, the significance of these properties for the full gravitational theory has remained unclear. There is a version of the CDJ ansatz in the real theory [13], but the remaining Gauss law constraints are considerably more difficult. The real Chern-Simons term does not seem to play a special role (apart from being a generating functional for the $B$ field, $B^{a i}=\frac{1}{2} \eta^{a b c} F_{b c}^{i}$ like in the complex theory). This also implies that the solutions to the Hamiltonian related to knot invariants (obtained by a formal loop transform of the Chern-Simons term from the connection to the loop representation [14]) do not carry over to the real theory. The absence of these "nice" features from the real theory may lead one to wonder whether they may not go away also in the complex case once reality conditions are properly taken into account.

The (quantum) Hamiltonian $H^{\mathscr{C}}$ of the complex theory has a natural representation in terms of loop functions, since the components of the field strength $F$ both in the continuum [2] and on the lattice [15] can be obtained by considering infinitesimal planar Wilson loops. We will show below that a similar statement holds for the real Hamiltonian $H^{\mathbb{R}}$, i.e., in spite of its nonpolynomiality, the potential term has a natural representation on quantum loop states, at least in the lattice formulation. This makes the search for zero eigenvectors of $\hat{H}^{\mathrm{R}}$ accessible numerically. Clearly the potential term presents a computational complication, which however in the absence of any explicit solutions of $\hat{H}^{\mathbb{C}} \Psi=0$ seems to be a matter of degree rather than one of principle. The big advantage of the real formulation is the presence of a well-defined and unambiguous scalar product on Wilson loop functionals in the quantum theory, that induced by the Haar measure of $\mathrm{SU}(2)$.

The following discussion will take place within the discrete lattice framework, with occasional comments on a possible continuum formulation. Furthermore, we will focus on the discussion of the potential term, which is new with respect to previous treatments $[15,4,7]$. It may be worthwhile noticing that in the lattice approach, both the state space and the operators get regulated simultaneously, since they share the same support (in terms of lattice links); in discretizing the state space, we get a regularization of the Hamiltonian "for free."

Recall now the basic ingredients of the Hamiltonian lattice formulation for theories based on a space of connections [16]. Our lattice will be a cubic $N \times N \times N$ lattice, with periodic boundary conditions. The basic operators associated with each lattice link $l$ are in our case an SU(2)-link holonomy $\hat{V}$ (represented by multiplication by $V$ ), together with its inverse $\hat{V}^{-1}$, and a pair of canonical momentum operators $\hat{p}_{i}^{+}$and $\hat{p}_{i}^{-}$where $i$ is an adjoint index. The operator $\hat{p}_{i}^{+}(n, \hat{a})$ is based at the vertex $n$, and is associated with the link $l$ oriented in the positive $\hat{a}$ direction. By contrast, $\hat{p}_{i}^{-}\left(n+\hat{1}_{\hat{a}}, \hat{a}\right)$ is based at the vertex displaced by one lattice unit in the $\hat{a}$ direction, and associated with the inverse link $l^{-1}(\hat{a})=l(-\hat{a})$. The wave functions are elements of $\times{ }_{l} L^{2}(\mathrm{SU}(2), d g)$, with the product taken over all links, and the Haar measure $d g$. The basic commutators are

$$
\begin{gathered}
{\left[\hat{V}_{A}^{B}(n, \hat{a}), \hat{V}_{C}^{D}(m, \hat{b})\right]=0,} \\
{\left[\hat{p}_{i}^{+}(n, \hat{a}), \hat{V}_{A}{ }^{C}(m, \hat{b})\right]=-(i / 2) \delta_{n m} \delta_{\hat{a} \hat{b}} \tau_{i A}{ }^{B} \hat{V}_{B}{ }^{C}(m, \hat{b}),} \\
{\left[\hat{p}_{i}^{-}(n, \hat{a}), \hat{V}_{A}^{C}(m, \hat{b})\right]=-(i / 2) \delta_{n, m+1} \delta_{\hat{a} \hat{b}} \hat{V}_{A}^{B}(m, \hat{b}) \tau_{i B}{ }^{C},} \\
{\left[\hat{p}_{i}^{ \pm}(n, \hat{a}), \hat{p}_{j}^{ \pm}(m, \hat{b})\right]= \pm i \delta_{n m} \delta_{\hat{a} \hat{b}} \epsilon_{i j k} \hat{p}_{k}(n, \hat{a}),} \\
{\left[\hat{p}_{i}^{+}(n, \hat{a}), \hat{p}_{j}^{-}(m, \hat{b})\right]=0,}
\end{gathered}
$$

where $\epsilon_{i j k}$ are the structure constants of SU(2). Our normalization for the $\mathrm{SU}(2)$ generators $\tau_{i}$ is such that $\left[\tau_{i}, \tau_{j}\right]=\epsilon_{i j k} \tau_{k}$ and $A_{a}=A_{a}^{i} \tau_{i} / 2$. Taking into account the expansions

$$
\begin{gathered}
V_{A}{ }^{B}(\hat{b})=1_{A}{ }^{B}+a A_{b A}{ }^{B}+O\left(a^{2}\right), \\
p_{i}^{ \pm}(\hat{b})=a^{2} E_{i}^{b}+O\left(a^{3}\right)
\end{gathered}
$$

of the corresponding classical quantities for small lattice spacing $a$, one derives the expansion

$$
\begin{aligned}
-\frac{1}{2} \operatorname{Tr} & {\left[\tau_{i} V(n, \hat{b}) p_{j}^{ \pm}\left(n+\hat{1}_{\hat{b}}, \hat{c}\right) \tau_{j} V(n, \hat{b})^{-1}\right]-p_{i}^{ \pm}(n, \hat{c}) } \\
& =a^{3}\left(\partial_{b} E_{i}^{c}+\epsilon_{i j k} A_{b j} E_{k}^{c}\right)+O\left(a^{4}\right)=a^{3} \nabla_{b} E_{i}^{c}+O\left(a^{4}\right) .
\end{aligned}
$$

The prefactor $-1 / 2$ occurs because of $\operatorname{Tr} \tau_{i} \tau_{j}=-2 \delta_{i j}$.

For reasons of symmetry we will from now on use the averaged momenta $p_{i}:=\left(p_{i}^{+}+p_{i}^{-}\right) / 2$ and their quantum versions. Motivated by Eq. (7), we may represent the quantum covariant derivative $\hat{\nabla}_{b} E_{i}^{c}$ by the lattice operator

$-\frac{1}{2} \operatorname{Tr}\left[\tau_{i} V(n, \hat{b}) \hat{p}_{j}\left(n+\hat{1}_{\hat{b}}, \hat{c}\right) \tau_{j} V(n, \hat{b})^{-1}\right]-\hat{p}_{i}(n, \hat{c})$.

Note, however, that this operator is well defined only on those Wilson loop states that for each occupied link $l(n, \hat{c})$ have also the neighboring "parallel" link $l\left(n+\hat{1}_{\hat{b}}, \hat{c}\right)$ occupied. [A lattice Wilson loop is a gauge-invariant function of the form $\operatorname{Tr} V\left(l_{1}\right) V\left(l_{2}\right) \ldots V\left(l_{k}\right)$, with $\gamma=l_{1} \circ l_{2} \circ \ldots \circ l_{k}$ a closed loop of lattice links.]

This happens because the left-hand side of expression (7) is a finite difference. If one of $\hat{p}_{j}\left(n+\hat{1}_{\hat{b}}, \hat{c}\right)$ and $\hat{p}_{i}(n, \hat{c})$ but not the other vanishes on a state $\Psi$, the result of the action of Eq. (8) on $\Psi$ for small lattice spacing $a$ is of lower order in $a$ and diverges in the limit as $a \rightarrow 0$. In particular, loop states with only sparse intersections have a singular behavior under the action of the covariant derivative operator (8). Moreover, since we do not want to distinguish any particular direction on the lattice, we will, instead of Eq. (8), use the averaged version

$$
\begin{aligned}
& -\frac{1}{4}\left\{\operatorname{Tr}\left[\tau_{i} V(n, \hat{b}) \hat{p}_{j}\left(n+\hat{1}_{\hat{b}}, \hat{c}\right) \tau_{j} V(n, \hat{b})^{-1}\right]\right. \\
& \left.-\operatorname{Tr}\left[\tau_{i} V\left(n-\hat{1}_{\hat{b}}, \hat{b}\right)^{-1} \hat{p}_{j}\left(n-\hat{1}_{\hat{b}}, \hat{c}\right) \tau_{j} V\left(n-\hat{1}_{\hat{b}}, \hat{b}\right)\right]\right\} .
\end{aligned}
$$

A remark similar to the one made above concerning the allowed loop states applies to this operator as well.

To obtain a well-defined lattice operator $\hat{H}^{\text {pot }}$, we still must take care about the determinantal factor $(\operatorname{det} E)^{-2}$. 
As shown in $[9,17], \quad \operatorname{det} E=(1 / 3 !) \eta_{a b c} \epsilon^{i j k} E_{i}^{a} E_{j}^{b} E_{k}^{c}$ possesses a natural quantum lattice analogue $\hat{D}(n):=\frac{1}{6} \eta_{a b c} \epsilon^{i j k} \hat{p}_{i}(n, \hat{a}) \hat{p}_{j}(n, \hat{b}) \hat{p}_{k}(n, \hat{c})$. Since the latter is a self-adjoint operator, there exists a Hilbert space basis in which it is diagonal. For the gauge-invariant sector, this basis is most easily constructed in terms of so-called spin network states, certain (anti)symmetrized, real linear combinations of Wilson loop states.

A spin network associates a positive "occupation number' with each lattice link, which may be interpreted as counting the number of (unoriented) flux lines of basic spin$\frac{1}{2}$ representations along the link, and also keeps track of the way in which those flux lines can be contracted gaugeinvariantly at the vertices (see [18] for more details). A concrete way of constructing elements of the spin network basis is to begin with sets of Wilson loops with fixed occupation numbers and arbitrary intertwiners, and then select linearly independent sets of intertwiners at the vertices (which generally, in terms of a loop language, are still related by Mandelstam constraints).

In terms of such states, the diagonalization of the operators $\hat{D}(n)$ is reduced to the diagonalization within finitedimensional subspaces of the Hilbert space. In the resulting diagonal basis we can meaningfully define quantum representations of arbitrary functions of $(\operatorname{det} E)$ in terms of their eigenvalues. (Investigations of the spectrum of (two related but not identical versions of) the volume operator have been performed in $[17,19]$.) In particular, if we restrict ourselves to eigenstates with nonvanishing eigenvalues, we can quantize $(\operatorname{det} E)^{-2}$ on the lattice. There is no immediate analogue of this construction in the continuum, although one can define a quantized version of the classical volume function $\int \sqrt{|\operatorname{det} E|}[8]$. Since the naive local quantum operator $\hat{\operatorname{det}} E$ vanishes at all points of a loop state without intersections, $1 / \hat{\operatorname{det}} E$ is ill defined almost everywhere on a typical loop state. In the continuum there thus seem to be no good analogues of the lattice states with "volume everywhere." A way out of this may be to either use smeared-out wave functions and/or regularize the quantum operators appropriately. Alternatively, one may multiply the Hamiltonian $H^{\mathrm{R}}$ by a factor $(\operatorname{det} E)^{2}$. Classically, this changes the constraint algebra at most by terms proportional to the constraints. In particular, the commutator of two Hamiltonians is just rescaled by a factor of $(\operatorname{det} E)^{4}$. Quantum-mechanically, however, operator ordering problems may appear.

Possibly the problem is not as serious as it seems since along smooth pieces of loop where $\hat{\operatorname{det}} E$ vanishes, also $(\operatorname{det} E)^{2} \hat{H}^{\text {pot }}$ does, and one may be able to regularize the action of $\hat{H}^{\text {pot }}$ to a finite value. Another problem, also relevant to the lattice approach, is that $\hat{\operatorname{det}} E$ has many zerovolume eigenstates even at loop intersections [9,17]. Hence the question is whether one can consistently restrict the Hilbert space so that the action of $\hat{H}^{\text {pot }}$ is always well defined (recall that $\left[\hat{H}^{\mathrm{R}}, \hat{\operatorname{det}} E\right] \neq 0$ ). For example, no immediate problems arise if one chooses a factor ordering for $\hat{H}^{\text {pot }}$ with the $\operatorname{det} E$ terms to the right and discards all zero-volume states by hand.

To summarize: there is a well-defined regularized, selfadjoint operator expression for the real Hamiltonian (3) on the lattice, at least on a large subsector of the Hilbert space of gauge-invariant functions. This operator is subject to the usual ambiguities with regard to factor ordering and addition of higher-order terms in the lattice spacing $a$. For example, we may choose

$$
\begin{aligned}
\hat{H}^{\mathrm{R}, \operatorname{latt}}(n)= & -\epsilon_{i j k} \operatorname{Tr}\left[\hat{V}\left(n, P_{\hat{a} \hat{b}}\right) \tau_{k}\right] \hat{p}_{i}(n, \hat{a}) \hat{p}_{j}(n, \hat{b})-\eta_{a_{1} a_{3} a_{4}} \eta_{b_{1} b_{3} b_{4}}\left[\hat{p}_{k}\left(n, \hat{a}_{3}\right) \hat{p}_{l}\left(n, \hat{a}_{4}\right) \hat{p}_{m}\left(n, \hat{b}_{3}\right) \hat{p}_{n}\left(n, \hat{b}_{4}\right)\right. \\
& \left.-2 \hat{p}_{m}\left(n, \hat{a}_{3}\right) \hat{p}_{n}\left(n, \hat{a}_{4}\right) \hat{p}_{k}\left(n, \hat{b}_{3}\right) \hat{p}_{l}\left(n, \hat{b}_{4}\right)\right] \hat{p}_{k}\left(n, \hat{a}_{2}\right) \hat{p}_{m}\left(n, \hat{b}_{2}\right) \\
& \times \frac{1}{4} \operatorname{Tr}\left[\tau_{l} \hat{V}\left(n-\hat{1}_{\hat{a}_{2}}, \hat{a}_{2}\right)^{-1} \hat{p}_{s}\left(n-\hat{1}_{\hat{a}_{2}}, \hat{a}_{1}\right) \tau_{s} \hat{V}\left(n-\hat{1}_{\hat{a}_{2}}, \hat{a}_{2}\right)-\tau_{l} \hat{V}\left(n, \hat{a}_{2}\right) \hat{p}_{s}\left(n+\hat{1}_{\hat{a}_{2}}, \hat{a}_{1}\right) \tau_{s} \hat{V}\left(n, \hat{a}_{2}\right)^{-1}\right] \\
& \times \operatorname{Tr}\left[\tau_{n} \hat{V}\left(n-\hat{1}_{\hat{b}_{2}}, \hat{b}_{2}\right)^{-1} \hat{p}_{t}\left(n-\hat{1}_{\hat{b}_{2}}, \hat{b}_{1}\right) \tau_{t} \hat{V}\left(n-\hat{1}_{\hat{b}_{2}}, \hat{b}_{2}\right)-\tau_{n} \hat{V}\left(n, \hat{b}_{2}\right) \hat{p}_{t}\left(n+\hat{1}_{\hat{b}_{2}}, \hat{b}_{1}\right) \tau_{t} \hat{V}\left(n, \hat{b}_{2}\right)^{-1}\right] \hat{D}(n)^{-2}
\end{aligned}
$$

for the Hamiltonian localized around a vertex $n$. In Eq. (10), all spatial indices $\hat{a}, \hat{b}$, etc., are summed over, and $V\left(n, P_{\hat{a} \hat{b}}\right)$ is the holonomy associated with a plaquette loop in the $\hat{a}-\hat{b}$ plane.

Note that the Hamiltonian of metric gravity (also containing inverse powers of $\operatorname{det} g=|\operatorname{det} E|$ ) cannot be treated in a similar way. The construction above depended on (i) the reformulation of canonical gravity in terms of connection variables, hence, (ii) the possibility of choosing a gaugeinvariant Hilbert space of Wilson loops, therefore, (iii) the diagonalization of the operators $\hat{D}(n)$ in terms of spin network states, together with (iv) a natural regularization of the covariant derivative terms in $\hat{H}^{\text {pot }}$.

Having thus set the stage for a systematic investigation of the eigenvalue problem $\hat{H}^{\mathrm{R}, \text { latt }} \Psi=0$, we will now describe some technical problems that have to be addressed for its solution. Consider the action of a local lattice Hamiltonian $\hat{H}^{\mathrm{R} \text {,latt }}(n)$ on a spin network state $\Psi$. Since the momentum operators do not change the occupation numbers $j_{i}$ of links, it a priori looks as if this action would result in a set of loop states with $\Delta j_{i} \in\{0,+1,+2\}$, depending on the contributions $\hat{V}$ (link) to the various links in a neighborhood of $n$, coming from the kinetic and potential parts of the Hamiltonian. Unfortunately, life is not as simple.

Take, for example, the action of the polynomial part $\hat{O}$ of $\hat{H}^{\text {pot }}$ on a spin network state. Our computations show that the resulting terms generically do not form a set of states that combine in a simple way to give one (or a small number of) spin networks, because the operator action does not preserve the total (anti-)symmetry over link permutations of the spin 
network. The result must always be expressible as a unique linear combination of spin network states, but it turns out that this decomposition in general contains states whose occupation numbers differ from those of the original state $\Psi$ within a whole range of values. For instance, the action of $\hat{O}(n)$ on a state $\Psi$ with occupation number $j_{i}$ for some link $l_{i}$ based at the vertex $n$ may result in a sum of spin network states with $j_{i}$ 's taking any one of the positive values $j_{i}+4, j_{i}+2, j_{i}, j_{i}-2, \ldots$ Moreover, through retracings of the form $V\left(n^{\prime}, \hat{a}\right) V\left(n^{\prime}, \hat{a}\right)^{-1} \equiv 1$ occurring during the decomposition of $\hat{O} \Psi$ into independent spin networks, even links may be affected that where not acted on directly by $\hat{O}(n)$ in the first place. This reveals a somewhat unpleasant property of the spin network states which in a sense are "maximally nonlocal" (as opposed to sets of maximally localized Wilson loop functions that one may favor in certain gauge-theoretic applications [20]), especially in conjunction with our requirement of selecting only spin networks with nonzero volume at every vertex.

We therefore conclude that the investigation of the spectrum of $\hat{H}^{\mathrm{R} \text {,latt }}$ requires the presence of an efficient algorithm for generating independent spin network states and computing inner products of such states. We reckon that even in the discretized lattice version the spectral problem is sufficiently complicated so as to make further approximations necessary. Since we can calculate matrix elements of the Hamiltonian explicitly, we can neglect small contributions, depending on suitable perturbation parameters such as those characterizing the spin network states or related to the bare gravitational coupling constant $G$. More details on our investigation of these issues will appear elsewhere.
Coming back to the continuum theory, one can show that the linearized limit of the real connection formulation coincides with that of the complex one (cf. the treatment of usual Ashtekar gravity in [21]). In the real case, the linearized versions of the kinetic and potential terms in Eq. (3) become proportional to each other and add up to the expected result. Likewise, the large- $G$ limit, as, for example, discussed in [22], is unchanged. For the $G \rightarrow 0$ limit [23], this does not necessarily seem to be the case.

Our real treatment (or an appropriate continuous analogue) can be viewed as complementary to another approach that has recently been suggested for dealing with the complex version of the theory, namely, making use of a "generalized Wick transform" [24]. In this ansatz, one tries to define a transformation $\hat{W}=\exp \hat{C}$, with $C=(\pi / 2) \int K_{a}^{i} E_{i}^{a}$ between two Hilbert spaces where in one the Hamiltonian has the simple form $H^{E}=\epsilon^{i j k} E_{i}^{a} E_{j}^{b} F_{a b}^{k}$ and in the other the more complicated form of the real theory. As far as we understand, the difficulties in making the operator $\hat{W}$ well defined in the continuum quantum theory are roughly comparable to those of constructing the continuum Hamiltonian $\hat{H}^{\mathrm{R}}$. (Note that, like the phase space functional $C$, also the potential term $H^{\text {pot }}$ can be written in terms of Poisson commutators of the quantities $\int(\operatorname{det} E)^{-1 / 2} H^{E}, \int \sqrt{\operatorname{det} E}, A_{a}^{i}$ and $E_{i}^{a}$ ) However, even if these could be overcome, the problem of finding nontrivial solutions to the Wheeler-DeWitt equation would still remain.

The author is indebted to F. Barbero for numerous discussions on real connection gravity.
[1] A. Ashtekar, Phys. Rev. Lett. 57, 2244 (1986); Phys. Rev. D 36, 1587 (1987).

[2] C. Rovelli and L. Smolin, Nucl. Phys. B331, 80 (1990).

[3] A. Ashtekar et al., J. Funct. Anal. 135, 519 (1996).

[4] R. Loll, Nucl. Phys. B444, 619 (1995).

[5] J.F. Barbero G., Phys. Rev. D 51, 5507 (1995).

[6] S. Holst, Phys. Rev. D 53, 5966 (1966).

[7] K. Ezawa, Mod. Phys. Lett. A 11, 349 (1996).

[8] C. Rovelli and L. Smolin, Nucl. Phys. B442, 593 (1995); B456, 753(E) (1995).

[9] R. Loll, Phys. Rev. Lett. 75, 3048 (1995).

[10] B. Brügmann et al., Phys. Rev. Lett. 68, 431 (1992).

[11] R. Capovilla et al., Phys. Rev. Lett. 63, 2325 (1989).

[12] H. Kodama, Phys. Rev. D 42, 2548 (1990).

[13] J.F. Barbero G., Class. Quantum Grav. 12, L5 (1995).

[14] B. Brügmann et al., Nucl. Phys. B385, 587 (1992); Gen. Relativ. Gravit. 25, 1 (1993).
[15] P. Renteln and L. Smolin, Class. Quantum Grav. 6, 275 (1989).

[16] J.B. Kogut and L. Susskind, Phys. Rev. D 11, 395 (1975); J.B. Kogut, Rev. Mod. Phys. 55, 775 (1983).

[17] R. Loll, Nucl. Phys. B460, 143 (1996).

[18] J.B. Baez, Adv. Math. 117, 253 (1996); in The Interface of Knots and Physics, edited by L. Kauffman (AMS, Providence, 1996); C. Rovelli and L. Smolin, Phys. Rev. D 52, 5743 (1995).

[19] R. De Pietri and C. Rovelli, Phys. Rev. D 54. 2664 (1996).

[20] R. Loll, Nucl. Phys. B368, 121 (1992); B400, 126 (1993).

[21] A. Ashtekar and J. Lee, Int. J. Mod. Phys. D 3, 675 (1994).

[22] V. Husain, Class. Quantum Grav. 5, 575 (1988).

[23] L. Smolin, Class. Quantum Grav. 9, 883 (1992).

[24] T. Thiemann, Class Quantum Grav. 13, 1383 (1996); A. Ashtekar, Phys. Rev. D 53, 2865 (1996). 